\title{
Numerical analysis and thermal fatigue life prediction of solder layer in a SiC-IGBT power module
}

\author{
Dian-hao Zhang, Xiao-guang Huang*, Bin-liang Cheng, Neng Zhang \\ College of Pipeline and Civil Engineering, China University of Petroleum (East China), Qingdao, 266580, China. \\ buangxg@upc.edu.cn
}

\begin{abstract}
Limited by the mechanical properties of materials, silicon ( $\mathrm{Si}$ ) carbide insulated gate bipolar transistor (IGBT) can no longer meet the requirements of high power and high frequency electronic devices. Silicon carbide (SiC) IGBT, represented by SiC MOSFET, combines the excellent performance of $\mathrm{SiC}$ materials and IGBT devices, and becomes an ideal device for high-frequency and high-temperature electronic devices. Even so, the thermal fatigue failure of SiC IGBT, which directly determines its application and promotion, is a problem worthy of attention. In this study, the thermal fatigue behavior of SiC-IGBT under cyclic temperature cycles was investigated by finite element method. The finite element thermomechanical model was established, and stress-strain distribution and creep characteristics of the $\mathrm{SnAgCu}$ solder layer were obtained. The thermal fatigue life of the solder was predicted by the creep, shear strain and energy model respectively, and the failure position and factor of failure were discussed.
\end{abstract}

KEYwORDS. SiC-IGBT; Thermal cycle; Thermal fatigue life; Creep; Solder layer.

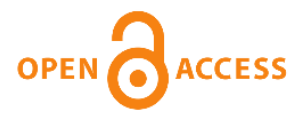

Citation: Zhang, D.H., Huang, X. G., Wang, Z. Q., Thermal fatigue analysis of the solder layer of SiC-IGBT power module, Frattura ed Integrità Strutturale, 55 (2021) 278-288.

Received: 23.10 .2020

Accepted: 22.12 .2020

Published: 01.01.2021

Copyright: (C) 2021 This is an open access article under the terms of the CC-BY 4.0, which permits unrestricted use, distribution, and reproduction in any medium, provided the original author and source are credited.

\section{INTRODUCTION}

7 he application in aerospace, automobile, oil drilling and other fields accelerates the improvement of electronic device of high power, high packaging density and high frequency. Under such working conditions, the reliability of solder layer in these electronic devices has become increasingly important. At present, the traditional silicon ( $\mathrm{Si}$ ) and gallium arsenide ( $\mathrm{GaAs}$ ) solders have been unable to meet the requirements of working environment characterized by high temperature, high-power and high-frequency, due to the limitations of the materials themselves. And their performances could not get a considerable progress from the manufacturing process or structural optimization [1-3]. Silicon carbide (SiC) material has better material properties has shown a broader prospect in electronic packaging, which is attributed to its high strength Si-C bonding [4]. Silicon carbide insulated gate bipolar transistors (SiC-IGBTs) are characterized by higher breakdown voltage, fast operating frequency, high power speed and high current density [5], therefore, they have better heat resistance than conventional Si-IGBTs and a wide potential application. At the same time, the highest operating junction temperature of the $\mathrm{SiC}-\mathrm{IGBT}$ can be as high as $175^{\circ} \mathrm{C}$, which allows the device itself be more adaptable to higher power density [6]. SiC-IGBT has become an ideal device in high-voltage and high-current applications, for instance, switching power supplies, AC motors, radar transmitters, inverters, and other power electronic devices [7]. 
It is proved that the most common failure mode in SiC-IGBT power modules, which was encapsulated by more than two SiC-IGBT semiconductor chips in the same substrate according to a certain circuit [8], is main caused by the interfacial cracking from the junction between solder and chip when undergoing alternating thermal loads or called thermal fatigue failure [9-10]. Therefore, it is significant to research the reliability of solder layer under the thermal cycles. Knecht and Fox [11] identified creep strain as the main cause of solder failure and proposed a model based on the creep strain range. Syed [12] investigated the development of a life prediction model for $\mathrm{SnAgCu}$ solders and predicted the fatigue life of solders under different intrinsic models using creep strain and creep energy dissipation density. Choi et al. [13] investigated the effect of different temperature changing frequencies on the life of IGBT power modules and established the relevant life factors, as well as the failure analysis of the tested IGBT modules. Zhu et al. [14] proposed a new creep-fatigue life model for solder joints at high strain rates, in which the creep damage and fatigue damage was calculated by Monkman-Grant equation and Coffin-Manson model, respectively. It was verified that the predictions were in good agreement with experimental results according to the Creep tests, and creep-fatigue tests performed. Elakkiya et al. [15] studied the effect of solder joint thickness on the service life of IGBT power semiconductors subjected to severe thermal stress. The results showed that creep strain occurred at the corner point of the solder layer and that the thinner the solder joint, the creep strain accumulated with temperature cycles. Samavatian et al. [16] found that the creep was the main failure mode of solder joints in the power semiconductors.

The reliability of SIC-IGBT power module is always threatened with the high operating temperature and high electric field strength in the switching process. It is of significance to carry out the structural analysis of the SiC-IGBT power module, and the results could provide certain advice for the optimization of SiC-IGBT package structure. Therefore, the thermomechanical finite element (FE) model under cyclic temperatures was established based on the creep constitutive model in this paper, and the cyclic stress and accumulated creep strain of $\mathrm{Sn}-\mathrm{Ag}$-Cu solder in the SiC-IGBT power module was estimated. As a result, the thermal fatigue of the SIC-IGBT power module was predicted from the perspective of creep strain and strain energy, and the main failure mode was discussed.

\section{NUMERICAL MODELLING}

\section{SiC-IGBT mode}

$\mathrm{F}$ igure 1 shows a schematic diagram of the SiC-IGBT cross-section, the layered structure are Cu base plate, base plate

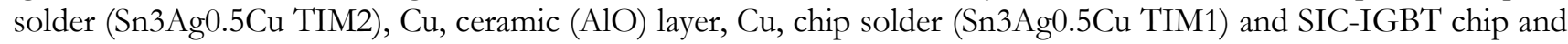
silica gel from bottom to top. In order to reduce the semiconductor losses in this process, a thick layer and low resistivity direct bonded copper-ceramic substrate is used which connects the chip to the substrate via a solder layer. Solder layer, which mainly achieve the link between the substrate and the chip by the reflow process, plays an important role in electrical connection between the chip and substrate and providing mechanical support for heat dissipation channels in the IGBT package module. Also, the solder layer should have a good thermal conductivity. At the same time, ultrasonic lead bonding technology is used to interconnect the chips and the external components. Efficient heat dissipation is achieved by the proper arrangement of the IGBT chip and the freewheeling diode.

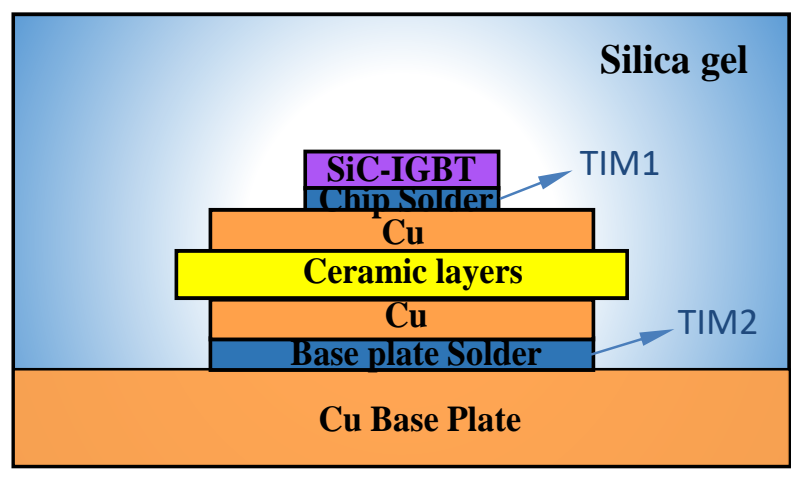

Figure 1: IGBT power module package structure diagram

During the operating state, frequent switching or external environment causes the internal temperature variation inside the SiC-IGBT power modules. Due to the mismatch of the coefficients of thermal expansion between silicon carbide, copper, ceramics, etc., and the geometrical constraint between each other in the package, the temperature change leads to the warp 
and warpback of the package body. As a visco-elastic intermediate layer, the solder layer always undergoes a great cyclic shear stress and creep strain, resulting in the thermal fatigue cracking of the solder layer. Therefore, the thermal fatigue behavior which caused by environment temperature has become a key issue in the reliability of SIC-IGBT power modules.

Creep constitutive model of $\mathrm{Sn} 3 \mathrm{Ag} 0.5 \mathrm{Cu}$.

According to the viscoelastic theory, the typical strain rate-stress relationship of Sn3Ag0.5Cu solder is linear at low stress, and power law creep at middle and high stresses. On the basis of the previous literature [17, 18], a hyperbolic sine power constitutive model is adopted, in which the relationship of strain rate with stress is linear at low stress and is hyperbolic sine power at the middle and high stresses, as shown in Eq. (1). At each temperature $T$, there exists a critical stress $\sigma_{\mathrm{v}}(T)$, which is used to separate the linear and power law creep stages. According to the creep results of two solder materials $\mathrm{Sn} 3 \mathrm{Ag} 0.5 \mathrm{Cu}$ and $\mathrm{Pb} 5 \mathrm{Sn}$, the strain rates under various stress levels and temperature-dependent $\sigma_{\mathrm{v}}$ are determined as follows:

$$
\dot{\varepsilon}= \begin{cases}A[\sinh (B \sigma)]^{n} \exp \left(-\frac{Q}{R T}\right) & \text { when } \sigma>\sigma_{v} \\ A_{0} \sigma \exp \left(-\frac{Q}{R T}\right) & \text { when } \sigma \leq \sigma_{v}\end{cases}
$$

in which

$$
\sigma_{v}=C_{0}-C_{1}\left(T-T_{R}\right)+C_{2}\left(T-T_{R}\right)^{2}
$$

where $\sigma_{v}$ is the linear creep limit, which is the cut-off point between linear and power creep, $T$ is the absolute temperature, $Q$ is the activation energy, $R$ is the universal gas constant, $\sigma$ is the equivalent stress, $T_{\mathrm{R}}$ is $273 \mathrm{~K}$. For $\mathrm{Sn} 3 \mathrm{Ag} 0.5 \mathrm{Cu}$ solder, $Q / \mathrm{R}=12993, n=5.85, B=0.145 \mathrm{MPa}^{-1}, C_{0}=17.357 \mathrm{MPa}, C_{1}=0.1219 \mathrm{MPa}^{-\mathrm{K}^{-1}}, C_{2}=2.457 \times 10^{-4} \mathrm{MPa} \mathrm{K}^{-2}, A=2.039 \times 10^{-4} \mathrm{~s}^{-1}$, and $A_{0}=2.039 \times 10^{-4} \mathrm{MPa}^{-1} . \mathrm{s}^{-1}[9]$.

\begin{tabular}{ccccccc}
\hline Material & $\begin{array}{c}\text { Elastic } \\
\text { modulus GPa }\end{array}$ & $\begin{array}{c}\text { Poisson's } \\
\text { ratio }\end{array}$ & $\begin{array}{c}\text { Coefficient of } \\
\text { thermal expansion } \\
10^{-6} / \mathrm{K}\end{array}$ & $\begin{array}{c}\text { Coefficient of heat } \\
\text { transfer W/(m.K) }\end{array}$ & $\begin{array}{c}\text { Specific Heat } \\
\mathrm{J} /(\mathrm{kg} \cdot \mathrm{K})\end{array}$ & $\begin{array}{c}\text { Density } \\
\mathrm{kg} / \mathrm{m}^{3}\end{array}$ \\
$\mathrm{Cu}$ & 110 & 0.34 & 16.4 & 398 & 385 & 8590 \\
$\mathrm{AlO}$ & 300 & 0.22 & 6.4 & 25 & 880 & 3800 \\
$\mathrm{SIC}$ chip & 400 & 0.14 & 4.2 & 150 & 700 & 3210 \\
Sn3Ag0.5Cu & 42.8 & 0.35 & 21.5 & 57 & 217 & 7390 \\
\hline
\end{tabular}

Table 1: The mechanical property of the SiC-IGBT power module.

\section{FE model of SiC-IGBT}

The finite element method is used to study the cyclic stress-strain behavior of a single-chip structure intercepted in the SiCIGBT power module during thermal cycles. Since the operating temperature of $\mathrm{SiC}$ is higher than that of Si material, the temperature cycle shown in Fig. 2 was adopted in the thermomechanical simulation of SiC-IGBT power module. In order to reduce the amount of calculation, 1/4 three-dimensional model, shown in Fig. 3, was established due to the structural symmetry of a single chip. A fixed constraint is applied at the bottom center point, and a symmetric boundary condition is applied to the symmetric plane. The sample is initially placed in a temperature field of $25^{\circ} \mathrm{C}$, and then the cycle temperature is applied to the surface of the power module. The size of the power module is as follows. The size of silicon carbide chip is $10 \times 10 \times 0.18 \mathrm{~mm}$, TIM1 is $10 \times 10 \times 0.1 \mathrm{~mm}$, the size of copper layer between the chip and DBC substrate is $15 \times 15 \times$ $0.3 \mathrm{~mm}$, the size of middle alumina is $17 \times 17 \times 0.38 \mathrm{~mm}$, the size of copper layer under middle alumina is $15 \times 15 \times 0.15 \mathrm{~mm}$, TIM2 is $15 \times 15 \times 0.2 \mathrm{~mm}$, and the size of the bottom copper substrate is $30 \times 30 \times 3 \mathrm{~mm}$, and the related material constants 
are shown in Tabs. 1-2. The symmetric constraints were applied in the $\mathrm{x}$ and $\mathrm{y}$ directions, and the $\mathrm{z}$ direction nodal displacement was restricted at the bottom surface of the model. The cyclic temperature was loaded at the exterior surface of the power module.

\begin{tabular}{cccccccc}
\hline $\begin{array}{c}\text { Temperature } \\
{ }^{\circ} \mathrm{C}\end{array}$ & $\begin{array}{c}\text { Elastic } \\
\text { modulus/ GPa }\end{array}$ & $\begin{array}{c}\text { Poisson's } \\
\text { ratio }\end{array}$ & $\begin{array}{c}\text { Coefficient of } \\
\text { thermal expansion } \\
10^{-6} / \mathrm{K}\end{array}$ & $\begin{array}{c}\text { Coefficient of } \\
\text { heat transfer } \\
\mathrm{W} /(\mathrm{m} . \mathrm{K})\end{array}$ & $\begin{array}{c}\text { Specific } \\
\text { Heat } \\
\mathrm{J} /(\mathrm{kg} \cdot \mathrm{K})\end{array}$ & $\begin{array}{c}\text { Density } \\
\mathrm{kg} / \mathrm{m}^{3}\end{array}$ & $\begin{array}{c}\text { Yield } \\
\text { strength }\end{array}$ \\
-75 & 54.311 & & 9.8 & & & 57.568 \\
-25 & 48.392 & & 15.9 & & & 41.253 \\
25 & 42.892 & \multirow{2}{*}{0.35} & 21.5 & 57 & 217 & 7390 & 29.00 \\
75 & 37.811 & & 22 & & & 20.82 \\
125 & 33.148 & & 22.7 & & & 16.98 \\
175 & 28.903 & & 23.6 & & & & \\
\hline
\end{tabular}

Table 2: Thermomechanical property of $\mathrm{Sn} 3 \mathrm{Ag} 0.5 \mathrm{Cu}$.

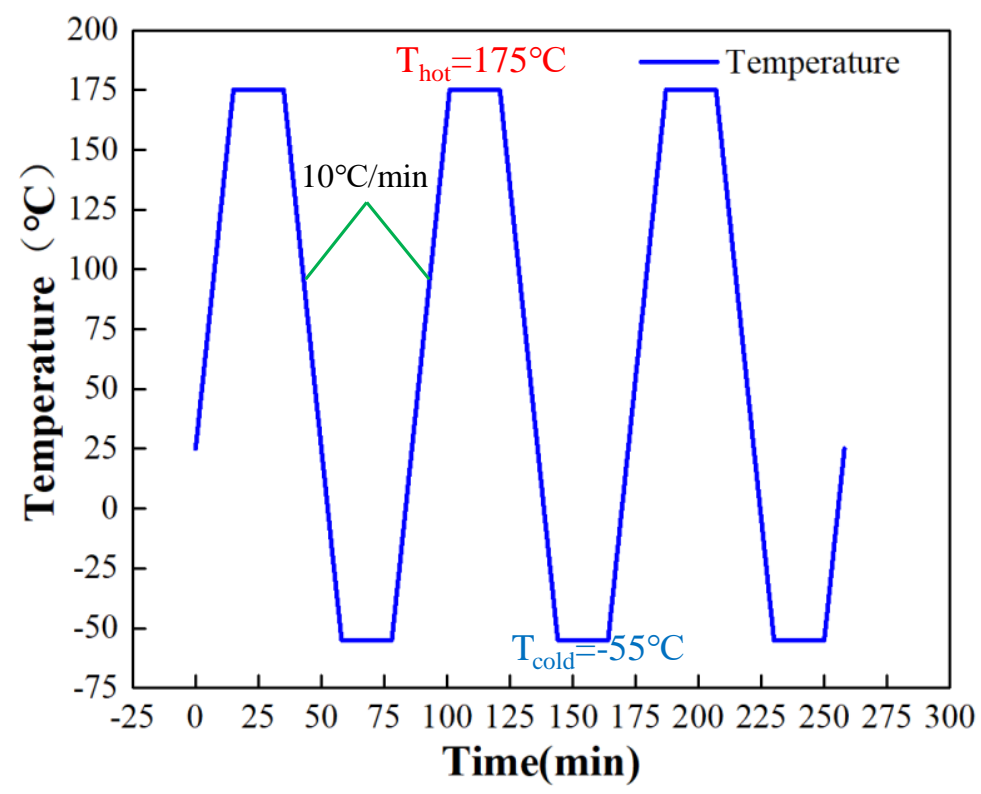

Figure 2: Cyclic temperatures in thermal fatigue

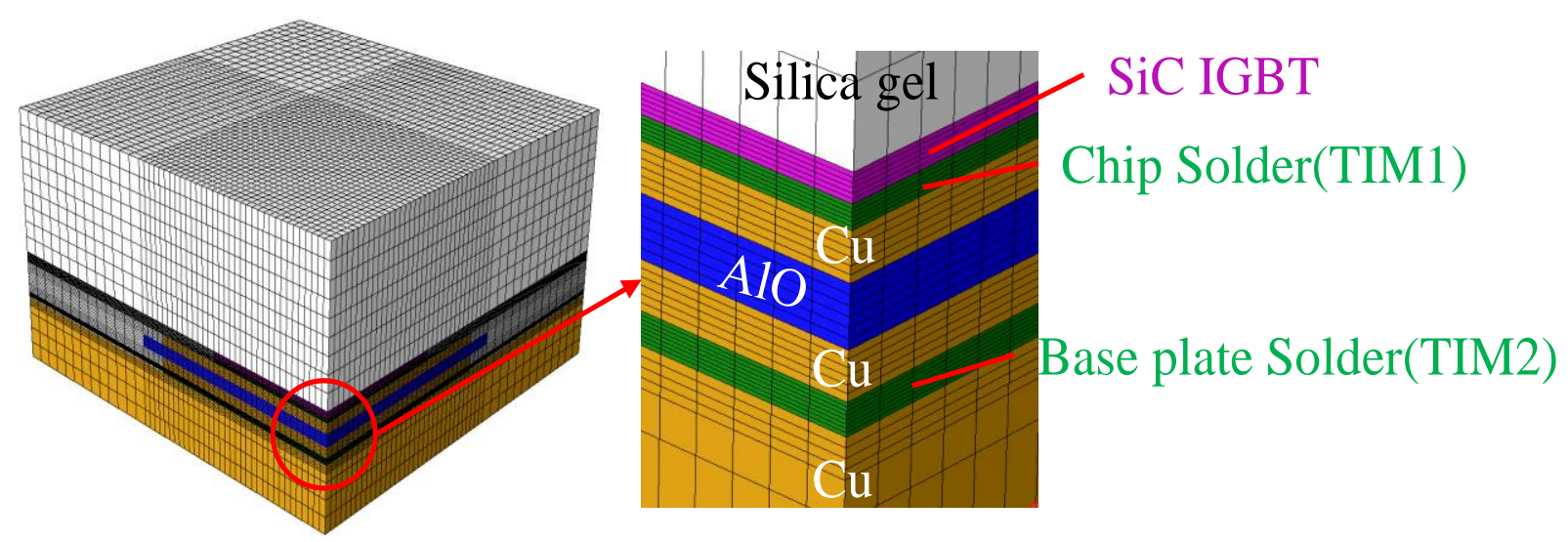

Figure 3: The 1/4 FE model 
The SiC-IGBT model was divided into a series of three dimensional 8-node reduced integration (C3D8R) elements. To select a suitable mesh density, we firstly conducted the reconstructed the convergence analysis of finite element model. Fig. 4 (a) -(c) illustrate the stress distribution in the solder layers with the coarse, medium and fine mesh, respectively. A comparison of the stresses shows that the results corresponding to the fine mesh presented in Fig. 4 are the most satisfactory. Therefore, this mesh density is selected in the following calculations, and the minimum mesh size at the interface of solder layer is refined to $0.01 \times 0.01 \times 0.002 \mathrm{~mm}$.

To verify the reliability of the finite element simulation, the IGBT in Xu's work [19], which has the same dimension, material properties and loading condition as our model and but different chip material ( $\mathrm{SiC}$ replaced by $\mathrm{Si}$ ) is established and the typical creep strain accumulation at the elements which has the maximum creep strain are demonstrated in Fig. 5 for comparison. It can be seen that the strain accumulations of TIM1 and TIM2 in our analysis has good agreement with Xu's result, indicating the analysis credibility of our finite element model.

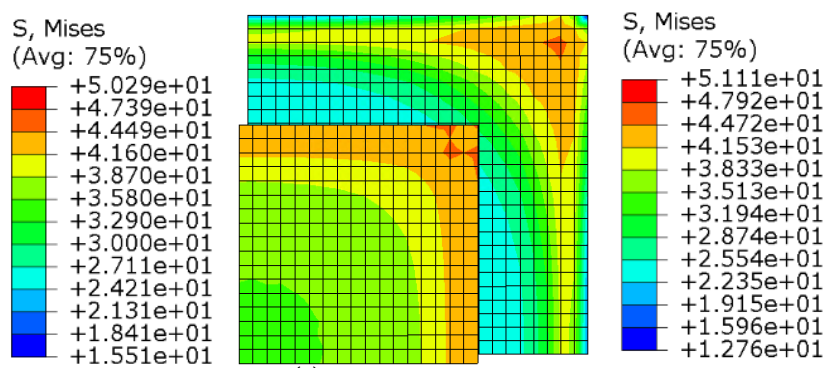

(a)

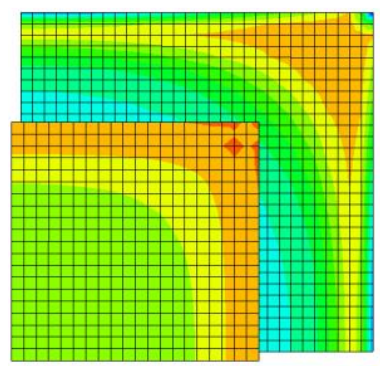

(b)

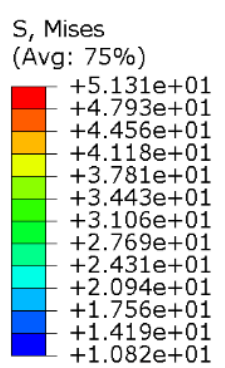

(c)

Figure 4: Convergence of stresses in the solder layers: (a) stress contours of coarse mesh, (b) stress contours of medium density mesh, (c) stress contours of fine mesh.

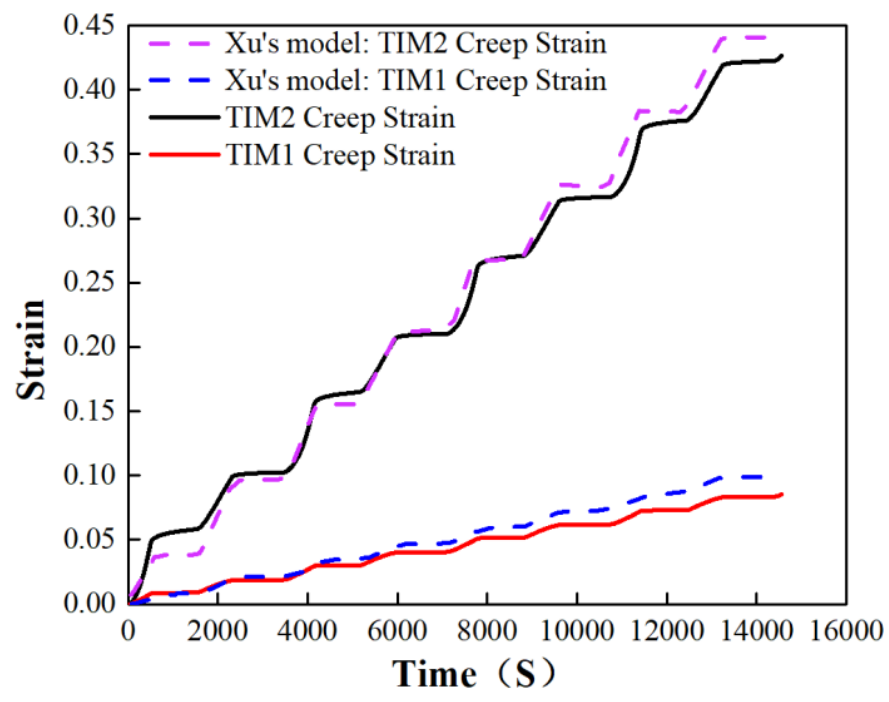

Figure 5: Accumulated creep strain

\section{FE analysis results}

Fig. 6 illustrates the overall temperature and Mises stress distribution of SiC-IGBT the maximum stress moment after ten thermal cycles. The maximum stress is located on the chip itself. However, due to creep properties and structural reasons, the failure of solder layer is what we focus on. Fig. 7 shows the Mises stress distribution in the TIM2 layer, the stresses at the corners are significantly higher than that in other positions. It may be caused by the interfacial effect in the solder layer near the interface. Although the maximum Mises stress in the solder does not exceed its yield strength which was showed in Tab. 2, the creep viscoelasticity that the material exhibits, will lead to the strain accumulation of elements in the solder layer. Once the accumulative creep strain exceed the critical strain, the element fails and the micro crack nucleates from the corner at the solder-chip or solder-substrate interface. The facts also proved that the thermal fatigue failure generally initiates from the corner of solder layer connected with the chip or substrate $[9,10]$. 

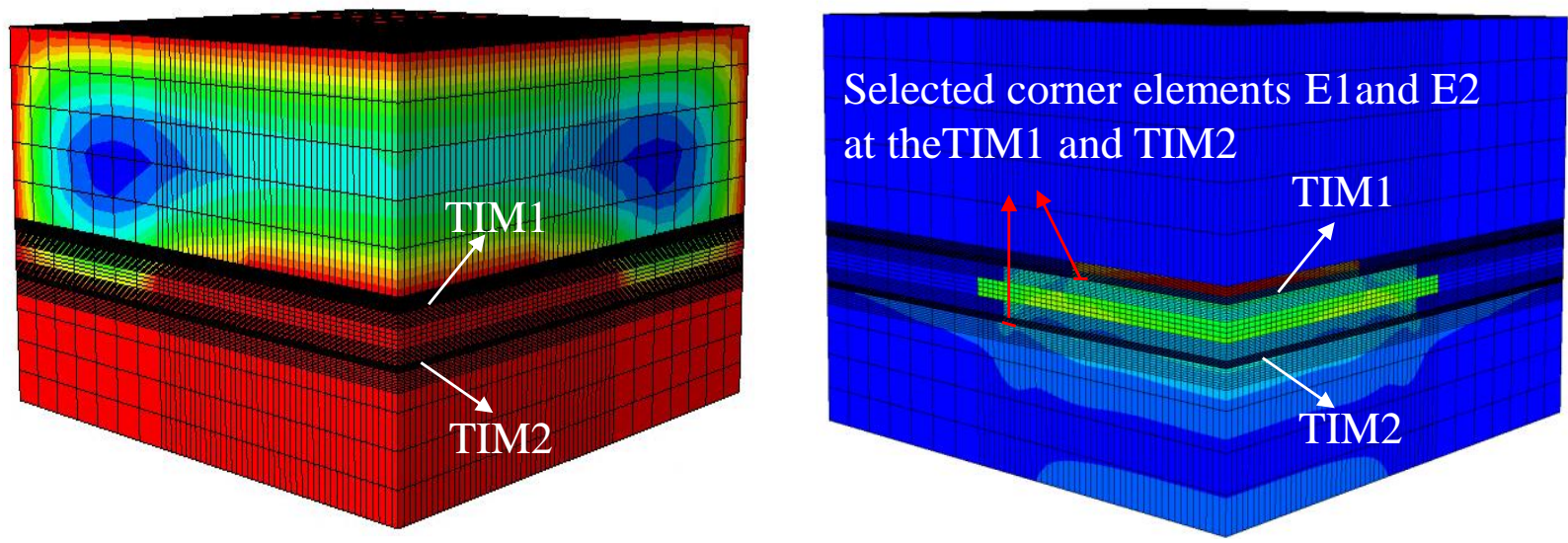

Figure 6: Stress distribution of 1/4 SiC-IGBT power module.
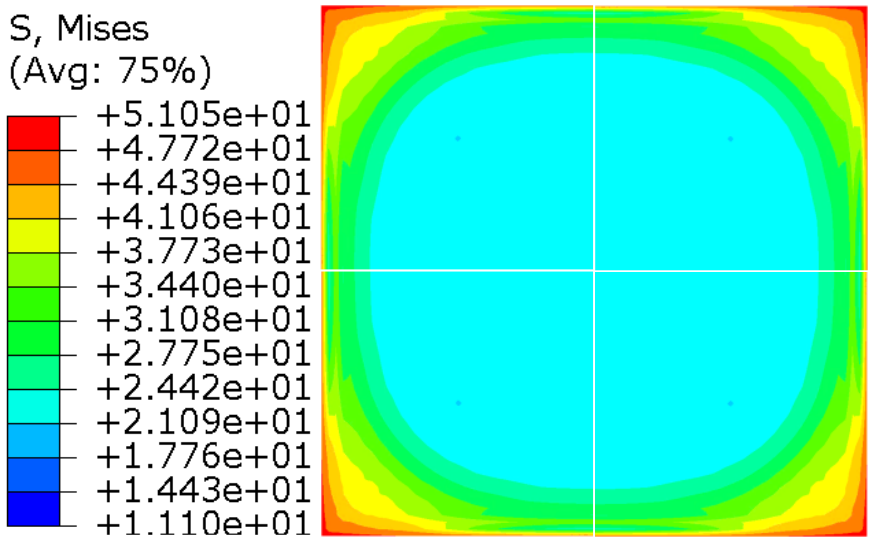

Figure 7: The stress distribution of TIM2 solder layer

Fig. 8 shows the temperature and Mises stress time history in the corner elements E1 and E2 at TIM1 and TIM2 layers, which are shown in Fig. 6, in first 10 temperature cycles. Due to the good thermal conduction capacity, the temperatures at elements E1 and E2 are almost same to the environmental temperature. There is a clear time lag between the stress history and environmental temperature history, which may attribute to the fact that it cost a little time for the creep strain of the solder accumulating to its maximum. The stress straights up at the heating process. Subsequently, the stress decreases nonlinearly due to stress relaxation and form a transient platform during the high temperature holding process. In the cooling stage, the stress decreases rapidly and reaches its minimum. It can be seen that the stress histories at the E1 and E2 are almost the same, although the maximum stress at E2 is relatively larger. TIM2 layer has a larger size and the stronger constraint on the deformation induced by thermal cycles, in other words, the effect of thermal deformation mismatch at TIM2 layer is more prominent.

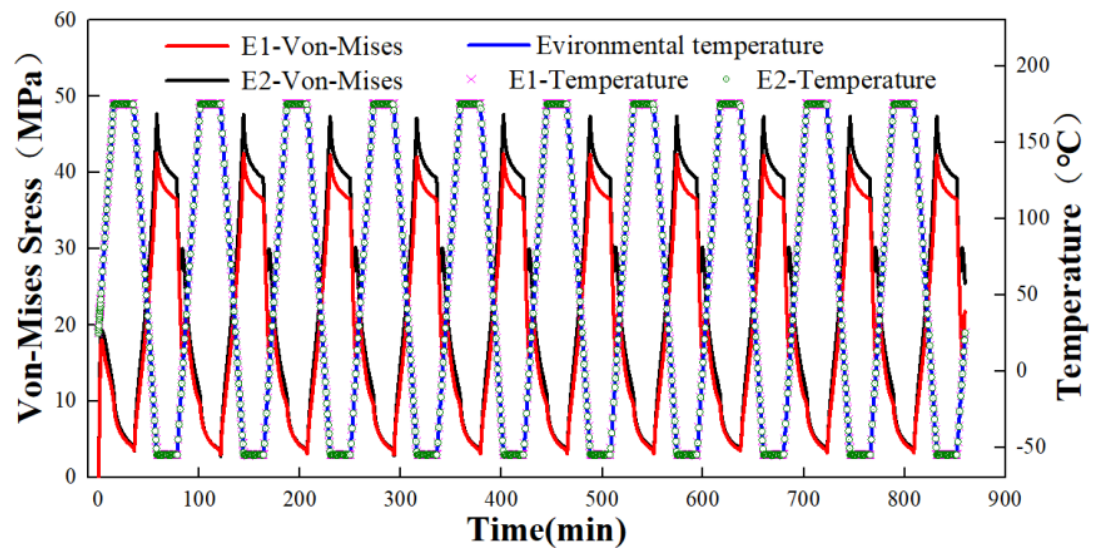

Figure 8: Mises stress time history of the elements E1 and E2. 


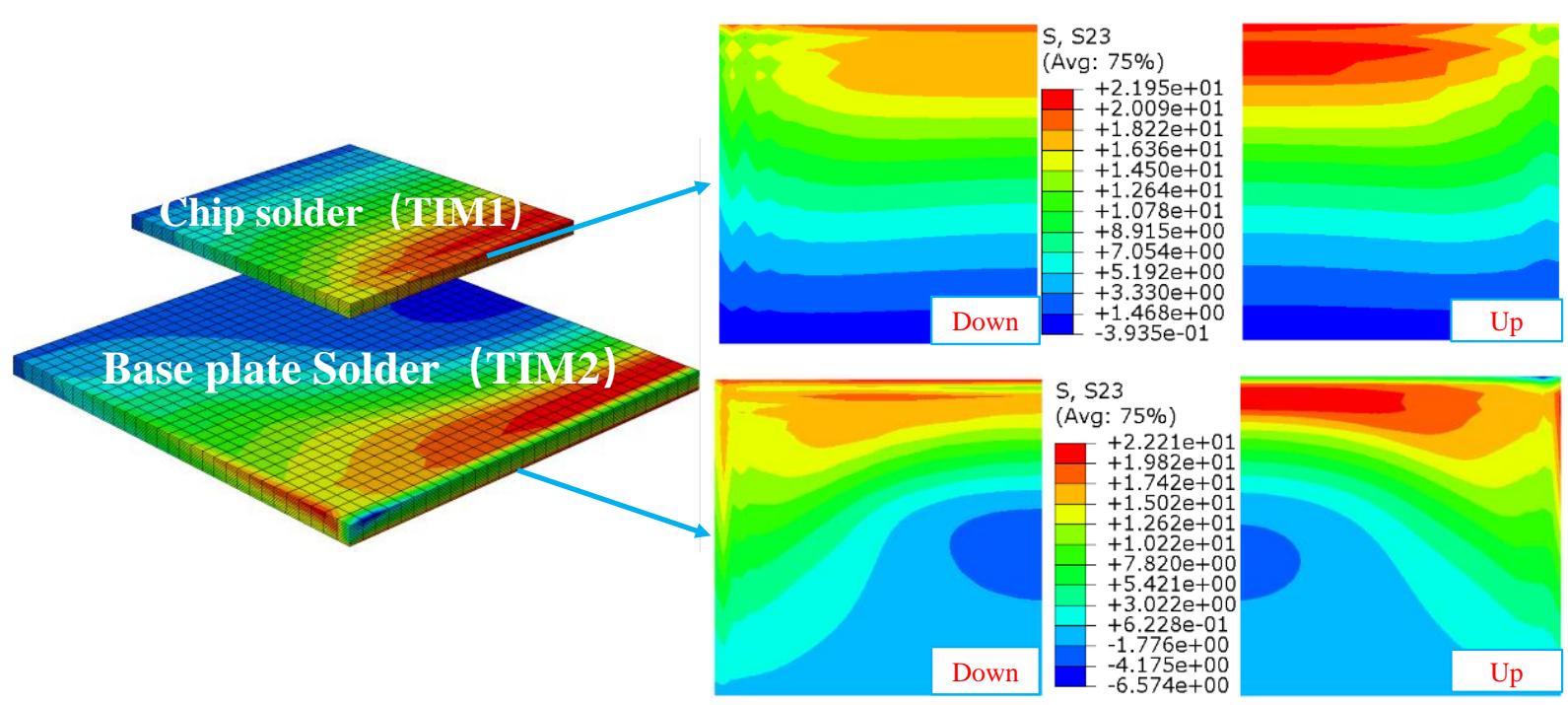

Figure 9: Shear stress distribution in the solder layer.

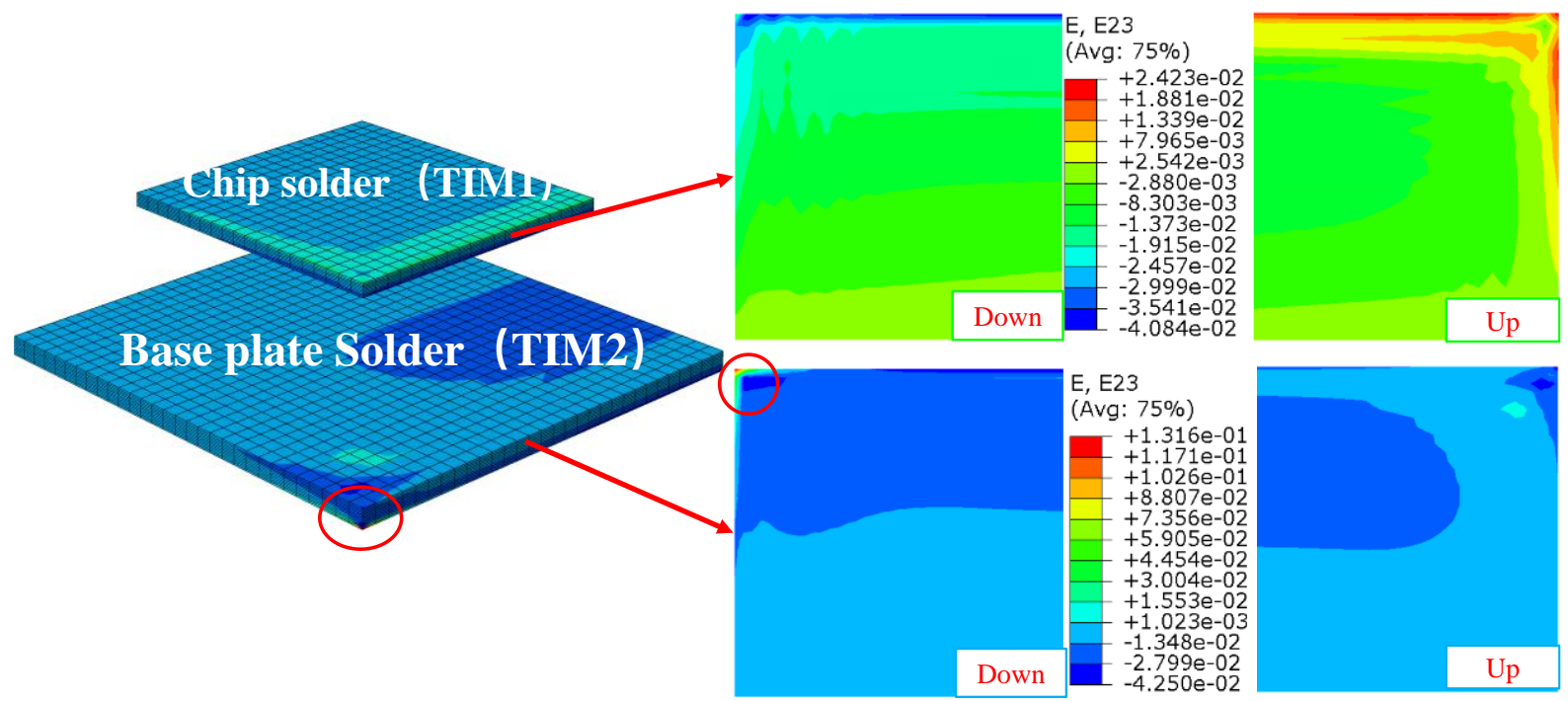

Figure 10: Shear strain distribution in the solder layer.

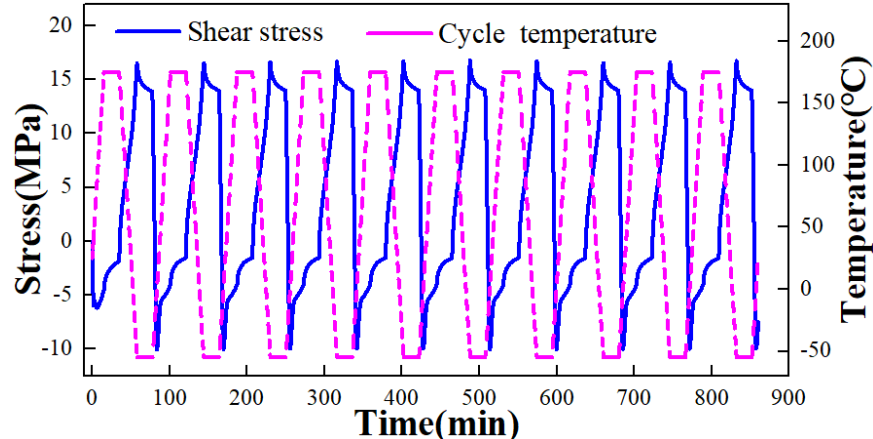

(a)

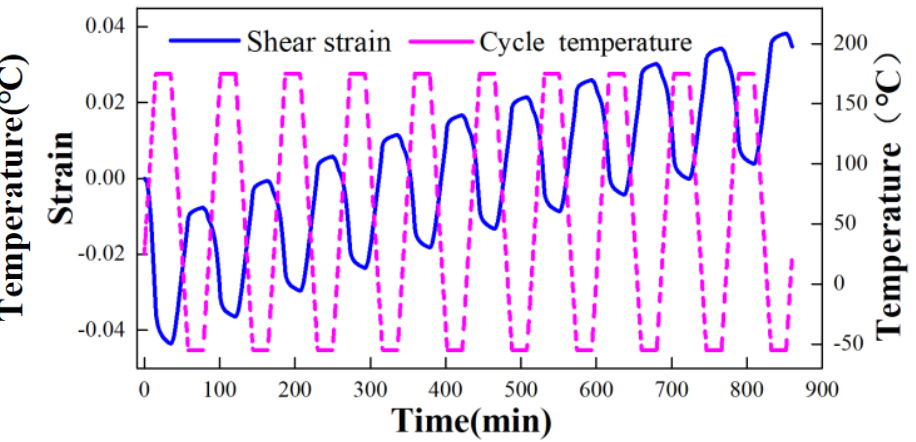

(b)

Figure 11: Shear stress-strain of element E2 at TIM2 solder layer: (a) stress, (b) strain.

Figs. 9-10 show the distribution of the maximum shear stress and shear strain of TIM1 and TIM2 layers, respectively. Whether in TIM1 and TIM2, the shear stress at the up surface is higher than that in the down surface, which may induce 
slight shear warpages in the solder layers. Although the maximum shear stress in TIM1 and TIM2 layers are about the same, the maximum shear strain in TIM2 is far higher than that in TIM1. The fact indicates that the SIC-IGBT may fail from TIM2 if the shear strain is the main failure mechanism.

Fig. 11 shows the time history of shear strain and shear stress of element E2 at TIM2 solder layer. Whether shear stress and shear strain exhibit good periodicities, which is consistent with the temperature cycle. The maximum shear stress in heating and cooling process both maintain unchanged. However, it is obvious that the shear strain gradually accumulates with the temperature cycles, which is related to the creep characteristics of the solder itself.

\section{FATIGUE LIFE PREDICTION}

A

number of thermomechanical fatigue life prediction models have been developed for the $\mathrm{Sn} 3 \mathrm{Ag} 0.5 \mathrm{Cu}$ solder joint [20], 17]. According to the creep mechanism, the Coffin-Manson based Engelmaier model [21], the accumulated creep strain based model and accumulated creep strain energy density based model, proposed by Syed [12, 24], were adopted to evaluate the thermal fatigue life, respectively. Engelmaier model considers the effect of frequency and temperature amplitude on fatigue life, and its formula follows:

$$
\mathrm{N}_{\mathrm{f}}=\frac{1}{2}\left(\frac{\Delta \gamma}{2 \varepsilon_{\mathrm{f}}}\right)^{1 / \alpha}
$$

where $N_{\mathrm{f}}$ is the fatigue life of the weld layer, $\Delta \Upsilon$ is the range of shear strain within one cycle, $\varepsilon_{\mathrm{f}}$ is the fatigue ductility coefficient of the solder, $\alpha$ is the fatigue ductility index of the solder related to the frequency and amplitude of cycling temperature, which is expressed as follows:

$$
\alpha=\lambda_{0}+\lambda_{1} \mathrm{~T}_{\mathrm{sj}}+\lambda_{2} \ln \left(1+\frac{360}{t_{\mathrm{dwell}}}\right)
$$

where $T_{\mathrm{sj}}$ is the average cyclical temperature of the solder layer, $t_{\text {dwell }}$ is the holding time of high and low temperature, $\lambda_{0}, \lambda_{1}$ and $\lambda_{2}$ are the material constants. For $S n 3 \mathrm{Ag} 0.5 \mathrm{Cu}$ solder, $\lambda_{0}=-0.367, \lambda_{1}=-9.69 \times 10^{-4}, \lambda_{2}=2.21 \times 10^{-2}$ [23].

Accordingly, the accumulated creep strain based life model could be described by the following equation:

$$
\mathrm{N}_{\mathrm{f}}=\left(\mathrm{C}^{\prime} \Delta \varepsilon_{\mathrm{acc}}\right)^{-1}
$$

where $\Delta \varepsilon_{\text {acc }}$ is the accumulated creep strain per cycle, $C^{\prime}$ is the inverse of creep ductility, $C^{\prime}=0.0405$ for $\operatorname{Sn} 3 \mathrm{Ag} 0.5 \mathrm{Cu}$ material . If the accumulated creep strain energy density is adopted as the key parameter which controls the thermal fatigue failure, the life prediction model could be simplified as

$$
\mathrm{N}_{\mathrm{f}}=\left(\mathrm{W}^{\prime} \Delta w_{\mathrm{acc}}\right)^{-1}
$$

where $\Delta w_{\text {acc }}$ is the accumulated creep strain energy density per cycle, $W /$ is the creep energy density for failure, $W^{\prime}=0.0014$ [22].

Fig. 12 shows the shear stress-strain hysteresis curve of element E2 at TIM2 solder layer. As the temperature cycles increases, the hysteresis curve gradually tends to coincide, i.e., the dissipated energy in every cycle reaches a steady state. The average shear strain range $\Delta \Upsilon$ of TIM2 is 0.0378 . Fig. 13 shows the creep strain and creep energy density curve of TIM2 solder layer. The creep strain and strain energy density increase gradually with temperature cycling. The calculated creep strain increment $\Delta \varepsilon_{\text {acc }}$ and strain energy increment $\Delta w_{\text {acc }}$ per cycle are 0.047 and $2.572 \mathrm{~J}$, respectively.

The predicted thermal fatigue life of TIM2 by different models are listed in Tab. 3, respectively, and the relative errors among three models are also listed for comparison. It can be seen that the predicted results from the Engelmaier model and creep strain energy density model are almost the same, and the thermal fatigue life from creep strain model is relatively shorter. But on the whole, the prediction results of these three models are acceptable. 


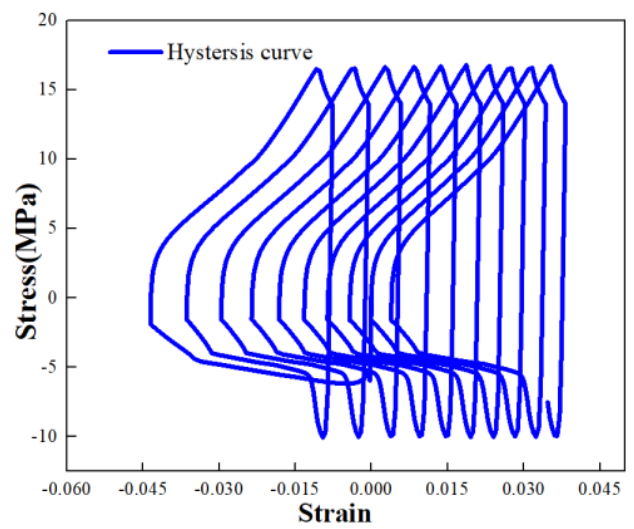

Figure 12: Cyclic stress/strain curves of solder layer TIM2

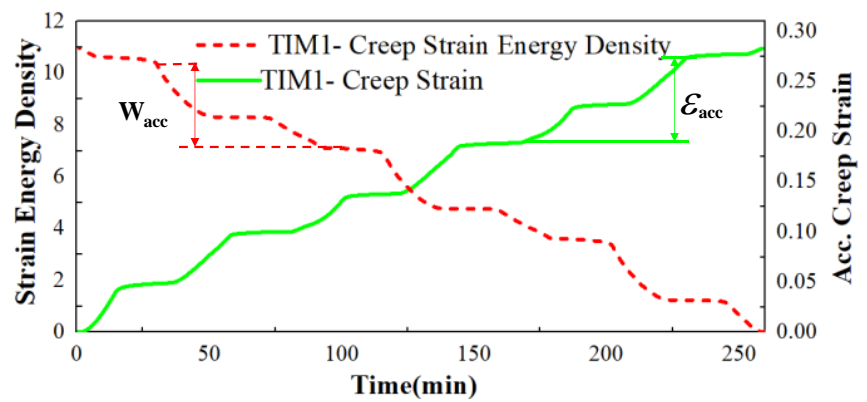

Figure 13: Accumulated creep strain and creep dissipation energy density distribution in solder join per cycle

\begin{tabular}{ccc}
\hline Theoretical model & Predicted results & Relative error $(\%)$ \\
Engelmaier model & 325 & 18.6 \\
Creep strain model & 274 & 17.0 \\
Creep strain energy density model & 330 & \\
\hline
\end{tabular}

Table 3: The predicted fatigue life of the TIM2 layer in SiC-IGBT power module.

\section{CONCLUSION}

$\mathrm{I}$ $\mathrm{n}$ this paper, the finite element thermomechanical model of SiC-IGBT power module was established, and the cyclical stress-strain distribution and creep behavior of the $\mathrm{Sn} 3 \mathrm{Ag} 0.5 \mathrm{Cu}$ solder layers was obtained under $-55^{\circ} \mathrm{C} \sim 175^{\circ} \mathrm{C}$ cyclic ambient temperature loading. Consequently, according to the obtained range of shear strain, accumulated creep strain and creep strain energy density at the corner point of the solder layer, the corresponding thermal fatigue lives were determined by Engelmaier model, creep strain model and creep strain energy density model, respectively. The comparisons of the predicted lives indicate that the thermal fatigue lives predicted are in an acceptable agreement. The proposed constitutive model of Sn3Ag0.5Cu solder and the FE-based thermal fatigue evaluation for the SIC-IGBT power module is feasible.

\section{ACKNOWLEDGMENT}

7 he research work was supported by the National Natural Science Foundation of China (No. 11972376), the National Natural Science Foundation of Shandong Province (No. ZR201910250083) and the Fundamental Research Funds for the Central Universities of China (No.20CX02308A). 


\section{REFERENCES}

[1] Han, L., Liang, L., Kang, Y., Qiu, Y. (2021). A review of SiC IGBT: models, fabrications, characteristics, and applications. IEEE Transactions on Power Electronics, 36(2), pp. 2080-93. DOI: 10.1109/TPEL.2020.3005940.

[2] He, P.Z., Zheng, L.B., Fang, H.C., Wang, C.L., Hua, J. (2013). Investigation of the temperature character of IGBT failure mode based the 3-D thermal-electro coupling FEM. Advanced Materials Research, 655-657, pp.1576-1580.

DOI: 10.4028/www.scientific.net/amr.655-657.1576.

[3] Ren, N., Hu, H., Lyu, X.F., Wu, J.P., Xu, H.Y., Li, R.G., Zuo, Z., Wang, K., Sheng, K. (2019). Investigation on single pulse avalanche failure of SiC MOSFET and Si IGBT. Solid-State Electronics, 152, pp. 33-40.

DOI: $10.1016 /$ j.sse.2018.11.010.

[4] Wright, N.G., Horsfall, A.B., Vassilevski, K. (2008). Prospects for SiC electronics and sensors. Materials Today, 11(12), pp.16-21. DOI:10.1016/S1369-7021(07)70348-6.

[5] Nawaz, M., Chimento, F. (2013). On the assessment of temperature dependence of 10-20 k V 4H-SiC IGBTs Using TCAD. Mater Sci Forum,740-742, pp.1085-8. DOI: 10.4028/www.scientific.net/MSF.740-742.1085.

[6] Madhusoodhanan, S., Mainali, K., Tripathi, A., Kadavelugu, A., Patel, D., Bhattacharya S. (2016). Power loss analysis of medium voltage three-phase converters using $15 \mathrm{kV} / 40$ A SiC N-IGBT. IEEE Journal of Emerging and Selected Topics in Power Electronics, 4(3), pp.901-917. DOI:10.1109/JESTPE.2016.2587666.

[7] Choi, U., Blaabjerg, F., Lee, K. (2015). Study and handing methods of power IGBT module failures in power electronic converter systems. IEEE Transaction on Power Electronics, 30 (5), pp.2517-2533. DOI:10.1109/TPEL.2014.2373390.

[8] Rashid, M. H. (2003). Power Electronics: Circuits, Devices, and Applications. Pearson Education India.

[9] Huang, X.G. (2019). Simulation on the interfacial singular stress-strain induced cracking of microelectronic chip under power on-off cycles. Journal of Microelectronics, Electronic Components and Materials, 49 (2), pp.69-77.

DOI: 10.33180/InfMIDEM2019.203.

[10] Huang, X.G., Wang, ZQ. (2020). Thermal fatigue evaluation model of a microelectronic chip in terms of interfacial singularity. Journal of Electronic Package, 142(011013), pp, 1-9. DOI: 10.1115/1.4045255.

[11] Knecht, S., Fox, L.R. (1990). Constitutive relation and creep-fatigue life model for eutectic tin-lead solder. IEEE Transactions on Components Hybrids and Manufacturing Technology, 13, pp.424-33. DOI: 10.1109/33.56179.

[12] Syed, A.R. (2004). Accumulated creep strain and energy density based thermal fatigue life prediction models for SnAgCu solder joints. In The 54th electronic components and technology conference, pp. 737-746.

[13] Choi, U-M., Blaabjerg, F., Jorgensen, S. (2017). Study on effect of junction temperature swing duration on lifetime of transfer molded power IGBT modules. IEEE Transactions on Power Electronics, 32(8), pp. 6434-6443. DOI: $10.1109 /$ TPEL.2016.2618917.

[14] Zhu, Y., Li, X., Wang C., Gao, R. (2015). A new creep-fatigue life model of lead-free solder joint. Microelectronics Reliability, 55(7), pp.1097-100. DOI: 10.1016/j.microrel.2015.03.019.

[15] Elakkiya, R., Kavithaa, G., Samavatian, V., Alhaifi, K., Kokabi, A., Moayedi, H. (2020). Reliability enhancement of a power semiconductor with optimized solder layer thickness. IEEE Transactions on Power Electronics, 35(6), pp.63976404. DOI: 10.1109/TPEL.2019.2951815.

[16] Samavatian, V., Iman-Eini, H., Avenas, Y., Samavatian, M. (2020). Effects of creep failure mechanisms on thermomechanical reliability of solder joints in power semiconductors. IEEE Transactions on Power Electronics, 35(9), pp. 8956-8964. DOI: 10.1109/TPEL.2020.2973312.

[17] Kim, J.W., Kim, D.G., Jung, S.B. (2006). Evaluation of displacement rate effect in shear test of Sn-3Ag-0.5Cu solder bump for flip chip application. Microelectronics Reliability, 46, pp.535-542. DOI: 10.1016/j.microrel.2005.06.008.

[18] Zhang, Y.M., Zhu, H.L., Fujiwara, M., Xu, J.Q., Dao, M. (2013). Low-temperature creep of SnPb and SnAgCu solder alloys and reliability prediction in electronic packaging modules. Scripta Materialia, 68, pp. 607-610.

DOI: 10.1016/j.scriptamat.2012.12.017.

[19] Xu, L. (2016). Research on reliability of IGBT power module packaging. Wuhan: Doctoral dissertation of Huazhong University of Science and Technology. (in Chinese)

[20] Wiese, S., Meusel, E. (2003). Characterization of lead-free solders in flip chip joints. Journal of Electronic Packaging, 125 (4), pp. 531-538. DOI:10.1115/1.1604155.

[21] Chauhan, P., Pecht, M., Osterman, M., Leer, S.W.R. (2009). Critical review of the Engelmaier model for solder joint creep fatigue reliability. IEEE Transactions on Components \& Packaging Technologies, 32(3), pp.693-700. 
DOI: $10.1109 /$ TCAPT.2009.2030983.

[22] Syed, A.R. (1997). ACES of finite element and life prediction models for solders and solder interconnections. In The symposium on the TMS conference, pp. 347-355.

[23] Chai, F., Osterman, M., Pecht, M. (2014). Strain-range-based solder life predictions under temperature cycling with varying amplitude and mean. IEEE Transactions on Device and Materials Reliability, 14(1), pp.351-357.

DOI: $10.1109 /$ TDMR.2013.2273121. 